\title{
THE
}

\section{Quantum and Classical Spin Clusters: Disappearance of Quantum Numbers and Hamiltonian Chaos}

\author{
Niraj Srivastava \\ Gerhard Müller \\ University of Rhode Island, gmuller@uri.edu
}

Follow this and additional works at: https://digitalcommons.uri.edu/phys_facpubs

Terms of Use

All rights reserved under copyright.

\section{Citation/Publisher Attribution}

Srivastava, N. \& Muller, G. (1990). Quantum and classical spin clusters: Disappearance of quantum numbers and Hamiltonian chaos. Zeitschrift für Physik B Condensed Matter, 81(1), 137-148.

Available at: http://dx.doi.org/10.1007/BF01454225 


\title{
Quantum and classical spin clusters: disappearance of quantum numbers and Hamiltonian chaos
}

\author{
Niraj Srivastava and Gerhard Müller \\ Department of Physics, University of Rhode Island, Kingston RI 02881, USA
}

\begin{abstract}
We present a direct link between manifestations of classical Hamiltonian chaos and quantum nonintegrability effects as they occur in quantum invariants. In integrable classical Hamiltonian systems, analytic invariants (integrals of the motion) can be constructed numerically by means of time averages of dynamical variables over phase-space trajectories, whereas in near-integrable models such time averages yield nonanalytic invariants with qualitatively different properties. Translated into quantum mechanics, the invariants obtained from time averages of dynamical variables in energy eigenstates provide a topographical map of the plane of quantized actions (quantum numbers) with properties which again depend sensitively on whether or not the classical integrability condition is satisfied. The most conspicuous indicator of quantum chaos is the disappearance of quantum numbers, a phenomenon directly related to the breakdown of invariant tori in the classical phase flow. All results are for a system consisting of two exchange-coupled spins with biaxial exchange and single-site anisotropy, a system with a nontrivial integrability condition.
\end{abstract}

\section{INTRODUCTION}

The concept of chaos in (mostly phenomenological) classical dynamical systems has received widespread attention and is being employed in an increasing number of different contexts. Manifestations of chaos in classical Hamiltonian dynamics [1-4] play a particularly important role in physics applications. Chaos is defined by the property of deterministic randomness. In nonintegrable classical Hamiltonian systems, deterministic randomness has its origin in the mixing character of the chaotic phase flow and is describable, for example, in terms of the Kolmogorov entropy. Chaotic phase-space trajectories are informationally incompressible and, therefore, (intrinsically or effectively) deterministically random. In their specification by a sequence of coordinates, redundancy is either absent (homoplectic situation) or irretrievable (autoplectic situation) [5-7].

In recent years, many quantum nonintegrability effects have been identified and studied [8-10]. The most prominent among them is perhaps the striking correlation between the fluctuation properties of the energy spectrum of a quantum model system and the (non-)integrability of the corresponding classical model [10-12]. However, there is at present no positive evidence for deterministic randomness in any property of nonintegrable quantum systems. A clearer picture of the still obscure connection between deterministic randomness and quantum mechanics is more and more urgently needed as experimental evidence for chaos in microscopic physical systems accumulates: microwave ionization measurements of highly excited hydrogen atoms $[13,14]$; photoabsorption spectroscopy of highly excited hydrogen atoms in strong static magnetic fields [15]; nonlinear dynamics of a pair of $\mathrm{Ba}^{+}$ions in a laser cooled trap [16].

Classical spin clusters and their well-defined quantum counterparts lend themselves ideally to detailed studies of quantum manifestations of Hamiltonian chaos. The present paper is, in fact, the fourth part of our study of the dynamics of integrable and nonintegrable spin clusters. In the first three parts, the focus was, respectively, on the following properties of classical 2-spin clusters: integrability criteria and analytic structure of invariants [17]; geometric structure of analytic and nonanalytic invariants [18]; time-dependent correlation functions and spectral properties [19, 20].

\section{THE 2-SPIN CLUSTER}

We consider a system of two localized classical 3component spins of fixed length $s$,

$$
\begin{aligned}
\mathbf{S}_{l} & =\left(S_{l}^{x}, S_{l}^{y}, S_{l}^{z}\right) \\
& =s\left(\sin \vartheta_{l} \cos \varphi_{l}, \sin \vartheta_{l} \sin \varphi_{l}, \cos \vartheta_{l}\right),
\end{aligned}
$$

$l=1,2$, specified by the energy function,

$$
H=\sum_{\alpha=x, y, z}\left\{-J_{\alpha} S_{1}^{\alpha} S_{2}^{\alpha}+\frac{1}{2} A_{\alpha}\left[\left(S_{1}^{\alpha}\right)^{2}+\left(S_{2}^{\alpha}\right)^{2}\right]\right\} .
$$

The dynamics is governed by the equations of motion,

$$
\frac{\mathrm{d} \mathbf{S}_{l}}{\mathrm{~d} t}=-\mathbf{S}_{l} \times \frac{\partial H}{\partial \mathbf{S}_{l}}=\left\{H, \mathbf{S}_{l}\right\}, \quad l=1,2,
$$

where $\left\{S_{l}^{\alpha}, S_{l^{\prime}}^{\beta}\right\}=-\delta_{l l^{\prime}} \sum_{\gamma} \epsilon_{\alpha \beta \gamma} S_{l}^{\gamma}$ are the Poisson brackets for classical spin variables. The corresponding quantum spin cluster is specified by the Hamiltonian

$$
\hat{H}=\hbar^{2} \sum_{\alpha=x, y, z}\left\{-J_{\alpha} \hat{\sigma}_{1}^{\alpha} \hat{\sigma}_{2}^{\alpha}+\frac{1}{2} A_{\alpha}\left[\left(\hat{\sigma}_{1}^{\alpha}\right)^{2}+\left(\hat{\sigma}_{2}^{\alpha}\right)^{2}\right]\right\}
$$

in terms of spin operators $\hat{\mathbf{S}}_{l}=\hbar \hat{\sigma}_{l}$ with spin quantum number $\sigma(\sigma=1 / 2,1,3.2, \ldots)$, which satisfy the commutation relations $\left[\hat{\sigma}_{l}^{\alpha}, \hat{\sigma}_{l^{\prime}}^{\beta}\right]=i \delta_{l l^{\prime}} \sum_{\gamma} \epsilon_{\alpha \beta \gamma} \hat{\sigma}_{l}^{\gamma}$. The classical spin model is obtained from its quantum counterpart as the limiting case [17],

$$
\hbar \rightarrow 0, \quad \sigma \rightarrow \infty, \quad \hbar \sqrt{\sigma(\sigma+1)}=s
$$


The operators $\hat{S}_{l}^{\alpha}$ whose mutual commutators vanish in that limit, can then be reinterpreted as the Cartesian components of the classical vector (II.1), and the Heisenberg equation of motion,

$$
\frac{\mathrm{d} \hat{\sigma}_{l}}{\mathrm{~d} t}=\frac{i}{\hbar}\left[\hat{H}, \hat{\sigma}_{l}\right], \quad l=1,2
$$

which governs the quantum time evolution, turns into Hamilton's equation of motion (II.3). Each classical spin $\mathbf{S}_{l}$ is expressible in terms of two canonical coordinates,

$$
p_{l}=\cos \vartheta_{l}, \quad q_{l}=\varphi_{l}
$$

The classical two-spin system (II.2) thus represents an autonomous Hamiltonian system of two degrees of freedom. Integrability of that system requires the existence of a $2^{\text {nd }}$ integral of the motion, $I\left(\mathbf{S}_{1}, \mathbf{S}_{2}\right)$, in addition to the energy function $H\left(\mathbf{S}_{1}, \mathbf{S}_{2}\right)$. In a previous study [17], we showed that with no single-site anisotropy, $A_{x}=A_{y}=A_{z}$, the 2 -spin system (II.2) is always integrable and the $2^{\text {nd }}$ analytic invariant has the form

$$
\begin{aligned}
I=- & \sum_{\alpha \beta \gamma=c y c l(x y z)} J_{\alpha} J_{\beta} S_{1}^{\gamma} S_{2}^{\gamma} \\
& +\sum_{\alpha=x y z} \frac{1}{2} J_{\alpha}^{2}\left[\left(S_{1}^{\alpha}\right)^{2}+\left(S_{2}^{\alpha}\right)^{2}\right] .
\end{aligned}
$$

Otherwise, the system is nonintegrable unless the interaction parameters satisfy the condition

$$
\begin{aligned}
& \left(A_{x}-A_{y}\right)\left(A_{y}-A_{z}\right)\left(A_{z}-A_{x}\right) \\
& +\sum_{\alpha \beta \gamma=\operatorname{cycl}(x y z)} J_{\alpha}^{2}\left(A_{\beta}-A_{\gamma}\right)=0,
\end{aligned}
$$

in which case the $2^{\text {nd }}$ integral of the motion is

$$
I=\sum_{\alpha=x y z} g_{\alpha} S_{1}^{\alpha} S_{2}^{\alpha}
$$

where

$$
\begin{aligned}
g_{\alpha} & =J_{\alpha}\left(J_{\alpha}+J_{\beta}+J_{\gamma}\right)+\left(A_{\alpha}-A_{\beta}\right) J_{\gamma}+\left(A_{\alpha}-A_{\gamma}\right) J_{\beta} \\
& -\left(A_{\alpha}-A_{\beta}\right)\left(A_{\alpha}-A_{\gamma}\right), \quad \alpha \beta \gamma=\operatorname{cycl}(x y z)
\end{aligned}
$$

\section{CLASSICAL INVARIANTS}

Integrability has far-reaching geometrical implications for the flow in the 4-dimensional phase space (in our case a compact manifold). Any phase point is constrained to move simultaneously on two independent 3-dimensional hypersurfaces,

$$
H\left(\mathbf{S}_{1}, \mathbf{S}_{2}\right)=\text { const }, \quad I\left(\mathbf{S}_{1}, \mathbf{S}_{2}\right)=\text { const. }
$$

The manifold which results from the intersection of these two hypersurfaces has the topology of a 2-dimensional torus (Poincaré-Hopf theorem). The phase space is densely foliated by such invariant tori. Each torus is specified by the values of two action variables $J_{1}, J_{2}$, determined by integrals

$$
J_{l}=\frac{1}{2 \pi} \sum_{k=1}^{2} \oint_{C_{l}} p_{k} \mathrm{~d} q_{k}, \quad l=1,2
$$

over two topologically independent closed paths $C_{l}$ on the torus. Consequently, the two invariants can be expressed as functions of two action variables:

$$
\begin{gathered}
H\left(\mathbf{S}_{1}, \mathbf{S}_{2}\right)=H^{\prime}\left(J_{1}, J_{2}\right), \\
I\left(\mathbf{S}_{1}, \mathbf{S}_{2}\right)=I^{\prime}\left(J_{1}, J_{2}\right) .
\end{gathered}
$$

In Ref. 18 we described a method for the numerical construction of invariants, a prescription applicable to quite general situations: Pick any dynamical variable $A$ which is independent of $H$ and determine its time average over the phase-space trajectory specified by initial condition $\left(\mathbf{S}_{1}, \mathbf{S}_{2}\right)$ :

$$
\langle A\rangle=\lim _{T \rightarrow \infty} \frac{1}{T} \int_{0}^{T} \mathrm{~d} t A\left(t ; \mathbf{S}_{1}, \mathbf{S}_{2}\right)=I_{A}\left(\mathbf{S}_{1}, \mathbf{S}_{2}\right) .
$$

According to the Birkhoff theorem [21], the function $I_{A}\left(\mathbf{S}_{1}, \mathbf{S}_{2}\right)$ is defined everywhere in phase space except for a set of points with measure zero, irrespective of whether the system is integrable or not. However, the properties of (III.4) depend sensitively on whether the phase flow is regular or chaotic.

\section{A. Analytic invariants}

For any integrable case of the 2-spin model (II.2), the two analytic invariants which guarantee integrability are the energy $E=H\left(\mathbf{S}_{1}, \mathbf{S}_{2}\right)$ and the function $I\left(\mathbf{S}_{1}, \mathbf{S}_{2}\right)$ given in (II.8) or (II.10) by analytic construction. In determining a function $I_{A}\left(\mathbf{S}_{1}, \mathbf{S}_{2}\right)$ from time averages (III.4) over single trajectories, we have to distinguish between rational tori (each containing a one-parameter family of periodic trajectories) and irrational tori (on which the phase flow is ergodic). Both sets of tori are dense in phase space; the rational ones have zero measure. If we restrict the initial conditions to points on irrational tori, then the invariant (III.4) is, in fact, a function of two variables only, the two action coordinates $J_{1}, J_{2}$ which specify individual tori. It is this function $I_{A}^{\prime}\left(J_{1}, J_{2}\right)=I_{A}\left(\mathbf{S}_{1}, \mathbf{S}_{2}\right)$ which can then be identified as an analytic invariant, a valid replacement for the explicitly known integral of the motion (II.8) or (II.10). In Ref. 18 we have numerically verified for specific choices of $A$ and an integrable 2-spin model that the function $I_{A}\left(\mathbf{S}_{1}, \mathbf{S}_{2}\right)$ is a piecewise smooth function of the phasespace variables with the singularities attributable to separatrices in the phase flow [22].

Here we take a different perspective in visualizing classical analytic invariants. The goal is to facilitate direct comparison with quantum invariants. Consider the 
two analytic invariants $\sqrt{\left\langle M_{x}^{2}\right\rangle}, \sqrt{\left\langle M_{z}^{2}\right\rangle}$, where $M_{\mu}=$ $\left(S_{1}^{\mu}+S_{2}^{\mu}\right) / 2$, as determined from tame averages over irrational tori, and the energy $E$ as a third analytic invariant. Since only two invariants can be independent, there exists an "equation of state", $E=E\left(\sqrt{\left\langle M_{x}^{2}\right\rangle}, \sqrt{\left\langle M_{z}^{2}\right\rangle}\right)$, which is represented by a piecewise smooth surface in $\left(\sqrt{\left\langle M_{x}^{2}\right\rangle}, \sqrt{\left\langle M_{z}^{2}\right\rangle}, E\right)$-space. Every point on that surface is the image of a point in the action plane $\left(J_{1}, J_{2}\right)$.

For a demonstration of analytic invariants in this representation consider the $X Y$ model with exchange anisotropy,

$$
H_{\gamma}=-(1+\gamma) S_{1}^{x} S_{2}^{x}-(1-\gamma) S_{1}^{y} S_{2}^{y}
$$

as a special case of (II.2) which is integrable for arbitrary $\gamma$. In Fig. 1 we show the section at $E=0.2$ of the invariant-surface $E\left(\sqrt{\left\langle M_{x}^{2}\right\rangle}, \sqrt{\left\langle M_{z}^{2}\right\rangle}\right)$ for several values of $\gamma$. We have determined $\sqrt{\left\langle M_{x}^{2}\right\rangle}$ and $\sqrt{\left\langle M_{z}^{2}\right\rangle}$ via time average (III.4) for initial conditions chosen randomly on the energy hypersurface. All points indeed fall onto piecewise smooth lines as expected. The cusps in the sections for $\gamma \neq 0$ represents singularities in the (not explicitly known) functional dependence of the invariants $\sqrt{\left\langle M_{x}^{2}\right\rangle}, \sqrt{\left\langle M_{z}^{2}\right\rangle}$ on $J_{1}, J_{2}$.

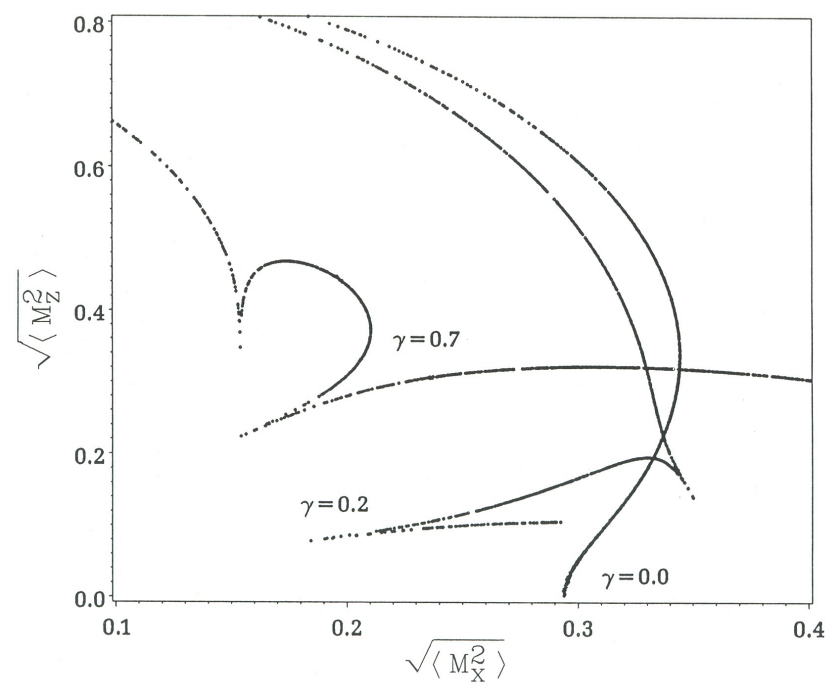

FIG. 1: Invariant $\sqrt{\left\langle M_{z}^{2}\right\rangle}$ versus invariant $\sqrt{\left\langle M_{x}^{2}\right\rangle}$ at energy $E=0.2$ for the cases $\gamma=0, \gamma=0.2$, and $\gamma=0.7$ of the classical 2-spin model $H_{\gamma}$ with $s=l$. The invariants are determined by time averages over individual trajectories for initial conditions randomly chosen on the energy hypersurface. The number of initial conditions used was 1200 for each line. The results of a small number of time averages were discarded because of extremely poor convergence.

\section{B. Nonanalytic invariants}

For nonintegrable cases of the 2-spin model (II.2) the phase flow is qualitatively different: All rational tori are destroyed and a fraction of the irrational ones as well. That part of the phase flow is converted into new tori (island chains) surrounded by chaotic phase flow. Within the chaotic flow there exist special types of nonchaotic trajectories: cantori (invariant Cantor sets) and a dense set of unstable periodic trajectories. The surviving irrational tori are no longer dense anywhere in phase space, but have nonzero measure, nevertheless. If we restrict our consideration of time averages to initial conditions corresponding to the two types of trajectories which together exhaust the measure of phase space, intact tori and chaotic trajectories, then we can infer the following properties of the function $I_{A}\left(\mathbf{S}_{1}, \mathbf{S}_{2}\right)$, which we now call a nonanalytic invariant: For initial conditions on intact tori, the invariant $I_{A}\left(\mathbf{S}_{1}, \mathbf{S}_{2}\right)$ again depends on two variables $J_{1}, J_{2}$ via the integrals (III.2), but they can no longer be interpreted as global action coordinates. The set of $\left(J_{1}, J_{2}\right)$-pairs for which the functions $H^{\prime}\left(J_{1}, J_{2}\right)$ and $I_{A}^{\prime}\left(J_{1}, J_{2}\right)$ are defined is no longer dense anywhere in the action plane. Canonical equations can therefore not be derived from $H^{\prime}\left(J_{1}, J_{2}\right)$. Nevertheless, in regions of phase space where intact tori are abundant, $I_{A}\left(\mathbf{S}_{1}, \mathbf{S}_{2}\right)$ exhibits a fairly smooth dependence on initial conditions, implying that the functions $H^{\prime}\left(J_{1}, J_{2}\right)$ and $I_{A}^{\prime}\left(J_{1}, J_{2}\right)$ are equally smooth on a coarse-grained scale. If it is assumed that the phase flow within any connected region of chaos is ergodic, then the function $I_{A}\left(\mathbf{S}_{1}, \mathbf{S}_{2}\right)$ on a line of initial conditions at constant $E$ consists, effectively, of horizontal steps of various sizes down to infinitesimal ones, a devil's staircase with steps up and down occurring for initial conditions located on intact tori [23].

For a representation of nonanalytic invariants by means of an equation of state, we consider the X Y model with single-site anisotropy,

$$
\begin{aligned}
H_{\alpha}= & -\left(S_{1}^{x} S_{2}^{x}+S_{1}^{y} S_{2}^{y}\right) \\
& -\frac{1}{2} \alpha\left[\left(S_{1}^{x}\right)^{2}-\left(S_{1}^{y}\right)^{2}+\left(S_{2}^{x}\right)^{2}-\left(S_{2}^{y}\right)^{2}\right],
\end{aligned}
$$

as a special case of (II.2) which is nonintegrable for $\alpha \neq 0, \pm 1$. Figure 2 shows the section at $E=0.2$ of the invariant-surface $E\left(\sqrt{\left\langle M_{x}^{2}\right\rangle}, \sqrt{\left\langle M_{z}^{2}\right\rangle}\right)$ for the integrable cases $\alpha=0$ and $\alpha=1$, and of its remnants for the nonintegrable case $\alpha=0.7$. As in Fig. 1, the data points represent time averages (III.4) for initial conditions randomly chosen on the energy hypersurface. The letters (a), (b), (c) label 3 different types of invariant tori.

In the integrable limit $\alpha=0$, the entire energy hypersurface is densely foliated by type (b) tori, giving rise to a perfectly smooth invariant-surface as observed in Fig. 2. With e increasing from zero, type (a) tori make their appearance on the energy hypersurface, separated from type (b) tori by a separatrix in the form of a chaotic band of increasing width. Type (c) tori emerge from within that chaotic band and become quite prominent at $\alpha=0.7$. Upon further increase of $\alpha$, the chaotic band starts to shrink again and disappears in the integrable limit $\alpha=1$ along with the type (b) and type (c) tori, leaving a dense foliation of type (a) tori on the energy 


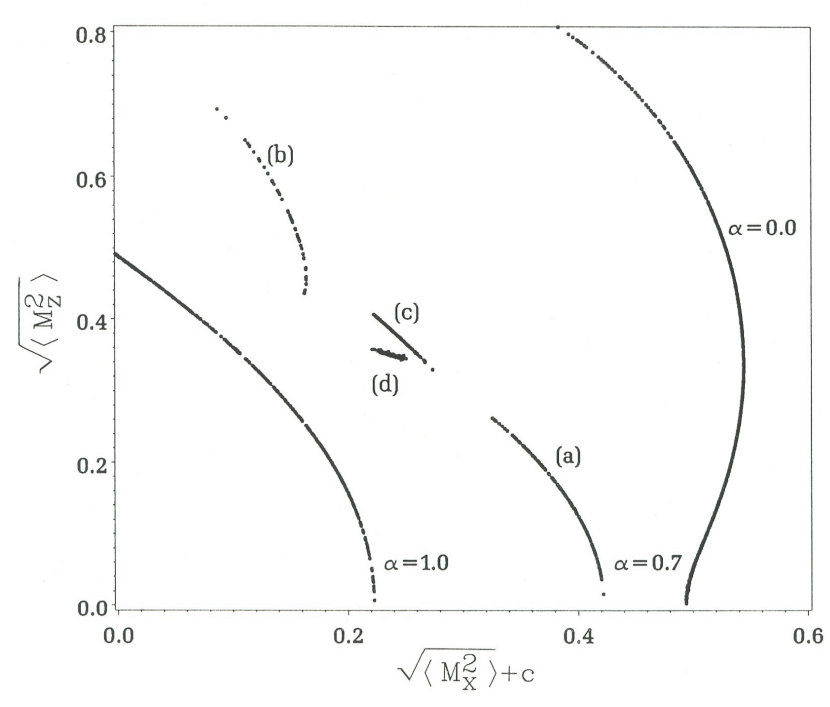

FIG. 2: Invariant $\sqrt{\left\langle M_{z}^{2}\right\rangle}$ versus invariant $\sqrt{\left\langle M_{x}^{2}\right\rangle}$ at energy $E=0.2$ for the cases $\alpha=0, \alpha=0.7$, and $\alpha=0.1$ of the classical 2-spin model $H_{\alpha}$ with $s=l$. For better display, the sections for $\alpha=0$ and $\alpha=1$ have been shifted horizontally by the amounts $c=0.2$ and $c=-0.2$, respectively. The invariants have been determined by time averages over individual trajectories for initial conditions randomly chosen on the energy hypersurface. The number of initial conditions chosen was 1200 for $\alpha=0,500$ for $\alpha=0.7$ and 600 for $\alpha=1$. The results of a small number of time averages were discarded because of extremely poor convergence.

hypersurface, resulting again in a perfectly smooth invariant surface.

For the nonintegrable case $\alpha=0.7$, we observe that parts of the invariant-surfaces pertaining to the integrable limits $\alpha=0$ and $\alpha=1$ are still visibly present at the top and bottom, respectively. These parts originate from phase-space regions in which, respectively, types (b) and (a) tori are predominant. However, no piece of invariant-surface no matter how small is perfectly smooth for $0<\alpha<1$, because of the omnipresence of chaotic trajectories. One interruption (separating the pieces marked (a) and (b)) occurs on a sufficiently large scale to be prominently displayed in Fig. 2. It is caused by the chaotic band mentioned previously, which is fairly widespread at $\alpha=0.7$.

For initial conditions within that chaotic region, the points $\left(\sqrt{\left\langle M_{x}^{2}\right\rangle}, \sqrt{\left\langle M_{z}^{2}\right\rangle}\right)$ tend to cluster at (d) near the center of the gap between the two major remnants of the invariant-surface. If it weren't for the slow convergence of the time averages along chaotic trajectories due to low-flux cantori [18-20], the entire chaotic region would be represented by a single isolated point in the constant-energy section of the invariant-surface. In the full $\left(\sqrt{\left\langle M_{x}^{2}\right\rangle}, \sqrt{\left\langle M_{z}^{2}\right\rangle}, E\right)$-space, the points associated with chaotic regions form string-like objects. Also in the gap between the two residual parts of invariant-surface, some of the time averages $\sqrt{\left\langle M_{x}^{2}\right\rangle}, \sqrt{\left\langle M_{z}^{2}\right\rangle}$ form a new piece of invariant-surface at (c) which originates from initial conditions on secondary KAM tori (island chain). This piece of invariant-surface has no smooth counterpart in any of the integrable limits. The characteristic pattern of invariant-surface interrupted by gaps, and the gaps populated with invariant-strings and new pieces of invariant-surface repeats itself on smaller scales ad infinitum. We shall demonstrate that the distinction between analytic and nonanalytic invariants, which characterizes integrable and nonintegrable classical Hamiltonian systems, can be translated into quantum mechanics along with many of its implications.

\section{QUANTUM INVARIANTS}

Consider the general quantum 2-spin system (II.4). If the model parameters satisfy the classical integrability condition (II.9), the quantum spectrum $E_{\lambda}$ is naturally catalogued as a two-parameter family in terms of two quantum numbers $m_{1}, m_{2}$, each representing one of two action variables,

$$
J_{k}=m_{k} \hbar, \quad m_{k}=-\sigma,-\sigma+1, \ldots, \sigma
$$

as prescribed by semiclassical quantization. For a given sequence of energy eigenvalues $E_{\lambda}$ resulting from the diagonalization of the Hamiltonian $\hat{H}$, the correct assignment of quantum numbers $m_{1}, m_{2}$ requires, in principle, that we know the function $H^{\prime}\left(J_{1}, J_{2}\right)$ in (III.3). In those integrable cases of (II.2) for which no separable canonical coordinates have been found, the implicit two-dimensional order of the eigenvalue sequence $E_{\lambda}$, i.e. its labelling in terms of two quantized action variables $m_{1}, m_{2}$ can be recovered nevertheless: Consider the energy eigenvalues $E_{\lambda}$ and the eigenvalues $I_{\lambda}$ of the quantum invariant $\hat{I}$, the quantum version of $I\left(\mathbf{S}_{1}, \mathbf{S}_{2}\right)$. In a diagram $I_{\lambda}$ versus $E_{\lambda}$, the array of $(2 \sigma+1)^{2}$ points form a regular pattern, which, however, is likely to be visually distorted due to the generally complicated nonlinear dependence of $E$ and $I$ on $J_{1}, J_{2}$. But we do not, in fact, need to know the $2^{\text {nd }}$ integral of the motion $\hat{I}$ explicitly to carry out this scheme; we can, following an idea of Peres [24], always construct it via time averages similar to the procedure previously employed for the classical model [18]: Take any operator $\hat{A}$ with $[\hat{A}, \hat{H}] \neq 0$, which represents a dynamical variable independent of $\hat{H}$, and consider the matrix elements $\hat{A}(t)$ in the energy representation,

$$
\left\langle\lambda|\hat{A}(t)| \lambda^{\prime}\right\rangle=\left\langle\lambda|\hat{A}| \lambda^{\prime}\right\rangle \mathrm{e}^{i\left(E_{\lambda}-E_{\lambda^{\prime}}\right) t / \hbar} .
$$

Performing the time average eliminates all off-diagonal elements:

$$
\overline{\left\langle\lambda|\hat{A}(t)| \lambda^{\prime}\right\rangle} \equiv \lim _{T \rightarrow \infty} \frac{1}{T} \int_{0}^{T} \mathrm{~d} t\left\langle\lambda|\hat{A}(t)| \lambda^{\prime}\right\rangle=\langle A\rangle_{\lambda} \delta_{\lambda \lambda^{\prime}} .
$$


The $2^{\text {nd }}$ integral of the motion $\hat{I}_{A}$ is then represented by the matrix

$$
I_{A}^{(\sigma)}=\Lambda_{\sigma} \cdot\langle A\rangle \cdot \Lambda_{\sigma}^{-1}
$$

where $\Lambda_{\sigma}$ is the eigenvector matrix of $H^{(\sigma)}$ for a given spin quantum number $\sigma$. If degenerate energy levels occur, the eigenvectors in the invariant subspaces must be chosen such that all off-diagonal elements $\left\langle\lambda|\hat{A}| \lambda^{\prime}\right\rangle$ are zero. Hence, for every operator $\hat{A}$ which is not an invariant, $[\hat{A}, \hat{H}] \neq 0$, there exists, in any $(2 \sigma+1)$-dimensional representation, an operator $I_{A}^{(\sigma)}$ which is an invariant, $\left[I_{A}^{(\sigma)}, H^{(\sigma)}\right]=0$, and which can be constructed by (IV.24). This holds true for any spin- $\sigma$ case of the general quantum spin Hamiltonian (II.4), irrespective of whether the corresponding classical spin model is integrable or not. However, the properties of the operator $I_{A}^{(\sigma)}$ depend sensitively on whether or not the classical integrability condition (II.9) is satisfied. In the following, we shall investigate quantum invariants for situations of increasing complexity.

\section{INTEGRABLE MODEL, SEPARABLE COORDINATES}

Consider the classical 2-spin model with uniaxially symmetric exchange coupling,

$$
H=-J\left(S_{1}^{x} S_{2}^{x}+S_{1}^{y} S_{2}^{y}\right)-J_{z} S_{1}^{z} S_{2}^{z} .
$$

In this model, integrability is guaranteed by Noether's theorem. The time evolution is in general nonlinear and can be determined analytically for arbitrary values of $J, J_{z}$.

\section{A. Action-angle variables}

In terms of the canonical coordinates (II.7), the Hamiltonian (V.1) reads

$$
H=-J \sqrt{s^{2}-p_{1}^{2}} \sqrt{s^{2}-p_{2}^{2}} \cos \left(q_{1}-q_{2}\right)-J_{z} p_{1} p_{2}
$$

The linear point transformation

$$
\begin{array}{ll}
Q_{1}=q_{1}+q_{2}, & P_{1}=\left(p_{1}+p_{2}\right) / 2, \\
Q_{2}=q_{1}-q_{2}, & P_{1}=\left(p_{1}-p_{2}\right) / 2
\end{array}
$$

transforms it into

$$
\begin{aligned}
H= & -J \sqrt{s^{4}-2 s^{2}\left(P_{1}^{2}+P_{2}^{2}\right)+\left(P_{1}^{2}-P_{2}^{2}\right)^{2}} \cos Q_{2} \\
& -J_{z}\left(P_{1}^{2}-P_{2}^{2}\right),
\end{aligned}
$$

where the coordinate $Q_{1}$ is cyclic. Its conjugate coordinate

$$
P_{1}=M_{z}=\left(S_{1}^{z}+S_{2}^{z}\right) / 2=\mathrm{const}
$$

is the integral of the motion derived from Noether's theorem. $H$ is separable in these coordinates. The characteristic function $W\left(Q_{1}, Q_{2} ; M_{z}, E\right)$, which is the solution of the Hamilton-Jacobi equation

$$
H\left(Q_{2} ; \frac{\partial W}{\partial Q_{1}}, \frac{\partial W}{\partial Q_{2}}\right)=E,
$$

has the general form

$$
W\left(Q_{1}, Q_{2} ; M_{z}, E\right)=Q_{1} M_{z}+W_{2}\left(Q_{2} ; M_{z}, E\right) .
$$

Equation (V.6) is then a $1^{\text {st }}$ order ODE in one variable,

$$
\begin{aligned}
& -J \sqrt{s^{4}-2 s^{2}\left[M_{z}^{2}+\left(\frac{\mathrm{d} W_{2}}{\mathrm{~d} Q_{2}}\right)^{2}\right]+\left[M_{z}^{2}-\left(\frac{\mathrm{d} W_{2}}{\mathrm{~d} Q_{2}}\right)^{2}\right]^{2}} \\
& \times \cos Q_{2}-J_{z}\left[M_{z}^{2}-\left(\frac{\mathrm{d} W_{2}}{\mathrm{~d} Q_{2}}\right)^{2}\right]=E
\end{aligned}
$$

and its solution thus reduced to quadrature. Given the solution of (V.8), the two action variables are obtained from

$$
\begin{gathered}
J_{1}^{\prime}=\frac{1}{2 \pi} \oint P_{1} \mathrm{~d} Q_{1}=2 M_{z} \\
J_{2}^{\prime}=\frac{1}{2 \pi} \oint P_{2}\left(Q_{2} ; M_{z}, E\right) \mathrm{d} Q_{2}=J_{2}\left(M_{z}, E\right),
\end{gathered}
$$

where

$$
\begin{aligned}
& P_{1}=\frac{\partial W}{\partial Q_{1}}=M_{z}=\text { const } \\
& P_{2}=\frac{\partial W}{\partial Q_{2}}=\frac{\mathrm{d} W_{2}}{\mathrm{~d} Q_{2}}=P_{2}\left(Q_{2} ; M_{z}, E\right) .
\end{aligned}
$$

Substitution of $J_{1}^{\prime}, J_{2}^{\prime}$ for $P_{1}, P_{2}$ in the characteristic function, $\bar{W}\left(Q_{1}, Q_{2} ; J_{1}^{\prime} J_{2}^{\prime}\right)=Q_{1} J_{1}^{\prime}+\bar{W}_{2}\left(Q_{2} ; J_{1}^{\prime} J_{2}^{\prime}\right)$ yields the angle variables,

$$
\begin{aligned}
\theta_{1}^{\prime}=\frac{\partial \bar{W}}{\partial J_{1}^{\prime}} & =Q_{1}+\frac{\partial \bar{W}_{2}}{\partial J_{1}^{\prime}}=\theta_{1}^{\prime}\left(Q_{1}, Q_{2} ; J_{1}^{\prime}, J_{2}^{\prime}\right) \\
& =\omega_{1}\left(J_{1}^{\prime}, J_{2}^{\prime}\right) t+\theta_{1}^{0}, \\
\theta_{2}^{\prime}=\frac{\partial \bar{W}}{\partial J_{2}^{\prime}} & \left.=\frac{\partial \bar{W}_{2}}{\partial J_{2}^{\prime}}=\theta_{2}^{\prime} Q_{2} ; J_{1}^{\prime}, J_{2}^{\prime}\right)=\omega_{2}\left(J_{1}^{\prime}, J_{2}^{\prime}\right) t+\theta_{2}^{0},
\end{aligned}
$$

where $\omega_{i}\left(J_{1}^{\prime}, J_{2}^{\prime}\right)=\partial \bar{H} / \partial J_{i}^{\prime}, i=1,2$ and $\bar{H}\left(J_{1}^{\prime}, J_{2}^{\prime}\right)=$ $H\left(Q_{2} ; P_{1}, P_{2}\right)$. This completes the solution of the classical 2-spin model (V.1). A more practical path to an explicit solution uses noncanonical variables which reduce this 2-spin model to that of a particle moving in a quartic potential (see Appendix). The line integral (V.9b) is then conveniently evaluated as a time integral

$$
J_{2}^{\prime}\left(M_{z}, E\right)=\frac{1}{2 \pi} \int_{0}^{\tau_{2}} \mathrm{~d} t \dot{Q}_{2}(t) P_{2}(t),
$$

over one period, $\tau_{2}\left(M_{z}, E\right)$, of the functions $P_{2} 2(t)=$ $z(t), Q_{2}(t)=\arctan \zeta(t)$ given in the Appendix. Along with (V.9a) this establishes the relationship between the two integrals of the motion $M_{z}, E$ and the two action variables $J_{1}, J_{2}$ for use in semiclassical quantization. 


\section{B. Quantum invariants $\hat{H}, \hat{M}_{z}$}

For the quantum version of model (V.1),

$$
\hat{H}=-\hbar^{2}\left[J\left(\hat{\sigma}_{1}^{x} \hat{\sigma}_{2}^{x}+\hat{\sigma}_{1}^{y} \hat{\sigma}_{2}^{y}\right)+J_{z} \hat{\sigma}_{1}^{z} \hat{\sigma}_{2}^{z}\right]
$$

a natural choice for the $2^{\text {nd }}$ invariant is the magnetization operator,

$$
\hat{M}_{z}=\hbar\left(\hat{\sigma}_{1}^{z}+\hat{\sigma}_{2}^{z}\right) / 2,
$$

which is diagonal in the energy representation. In Fig. 3 we have plotted the eigenvalues $M_{\lambda}^{z}$ versus energy $E_{\lambda}$, $\lambda=1, \ldots,(2 \sigma+1)^{2}$, for the 441 eigenstates of (V.13) with $\sigma=10$. The five different diagrams represent results from a numerical diagonalization off $\hat{H}$ for exchange constants (a) $J_{z}=0, J=1$, (b) $J_{z}=0.5, J=1$, (c) $J_{z}=J=1$, (d) $J_{z}=1, J=0.5$, (e) $J_{z}=1, J=0$, respectively. In each case the points form a highly regular pattern as is expected for an integrable model. In the $X Y$ model $\left(J_{z}=0\right)$, the pattern is symmetric about the two
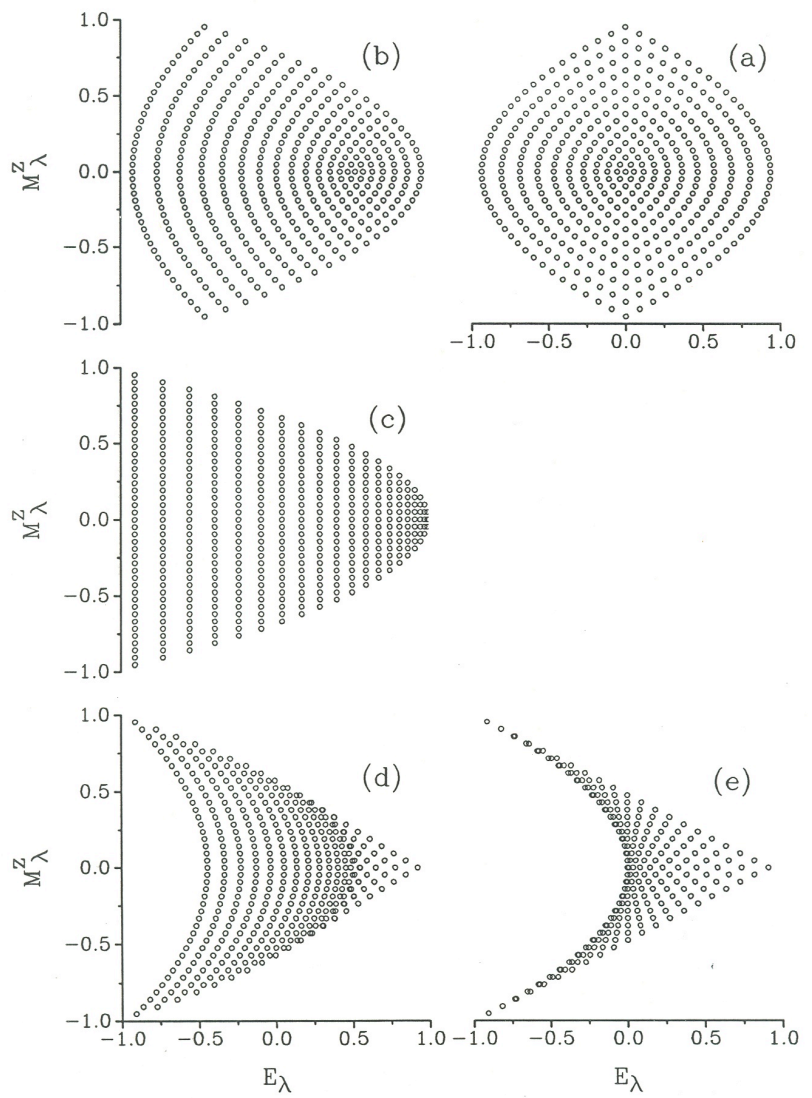

FIG. 3: Eigenvalue $M_{\lambda}^{z}$ (magnetization) versus eigenvalue $E_{\lambda}$ (energy) of all $(2 \sigma+1)^{2}=441$ eigenstates of the quantum 2-spin model (V.13) for $\sigma=10, s=1$ and parameter values (a) $J_{z}=0, J=1$, (b) $J_{z}=0.5, J=1$, (c) $J_{z}=J=1$, (d) $J_{z}=1, J=0.5$, (e) $J_{z}=1, J=0$. Data from a numerical diagonalization of $\hat{H}$. lines $M_{z}=0$ and $E=0$. There is no degeneracy of points in the $\left(E, M_{z}\right)$-plane. As $J_{z}$ increases from zero, the points move collectively. The pattern changes smoothly, maintaining the symmetry about the line $M_{z}=0$. Underlying this regular pattern and its gradual transformation is the existence of a one-to-one mapping between each array of points shown in Fig. 3 and the points on a perfect square lattice of size $(2 \sigma+1) \times(2 \sigma+1)$ representing the plane of quantized actions, a mapping which depends smoothly on the interaction parameters $J, J_{z}$. In the Heisenberg case $\left(J_{z}=J\right)$, the higher rotational symmetry of the model causes the points to line up in strings perpendicular to the E-axis. As $J_{z}$ increases beyond $J$, the movement of the points in the $\left(E, M_{z}\right)$-plane folds over at the edges of the pattern near the high-energy end. A twofold degeneracy of points in the plane starts to build up; it becomes complete and exact in the Ising $\operatorname{limit}(J=0)$.

The classical time evolution of the model (V.1) is nonlinear except for $J_{z}=J$ or $J=0$. As described in the Appendix, the meridional motion of the two classical spins maps onto the motion of a fictitious particle in a quartic potential. For $0<J_{z}<J$ that potential is a single well (in the form of an inverted double well). For $J_{z}=J$ it becomes harmonic, hence the linear time evolution. If $J_{z}>J$, the potential is a single well for small $E$ and double well for large $E$, precisely where the twofold degeneracy starts to build up in the quantum spectrum [Figs. 3 (d), (e)].

\section{Quantum numbers $m_{1}, m_{2}$}

According to (V.9) and (V.12), the dependence on $M_{z}, E$ of the two action variables $J_{1}^{\prime}, J_{2}$, for the model (V.1) can be expressed as

$$
J_{1}^{\prime}=2 M_{z}, \quad J_{2},=\frac{1}{2 \pi} \int_{0}^{\tau_{2}} \mathrm{~d} t \frac{z \dot{\zeta}}{1+\zeta^{2}}
$$

with explicit expressions for $z\left(t, M_{z}, E\right), \zeta\left(t, M_{z}, E\right)$ and $\tau_{2}\left(M_{z}, E\right)$ given in the Appendix. These exact expressions can now be used for the recovery of the quantum numbers associated with the action variables $J_{1}^{\prime}, J_{2}^{\prime}$ : Take the eigenvalues $E_{\lambda}, M_{\lambda}^{z}$ of the two quantum invariants from the numerically determined eigenstates of the quantum spin- $\sigma$ Hamiltonian (V.13), then evaluate the classical action integrals (V.15) for $E=E_{\lambda}, M_{z}=M_{\lambda}^{z}$, $\lambda=1, \ldots,(2 \sigma+1)^{2}$. According to semiclassical quantization, the values $J_{1}^{\prime}, J_{2}$, should fall, for $s=\hbar \sqrt{\sigma(\sigma+1)}$, onto the sites of a perfect square lattice with spacing $\hbar$.

Figure 4 depicts the nonlinear mapping $\left(M_{z}, E\right) \rightarrow$ $\left(J_{1}, J_{2}\right)$ for the case $J_{z}=0$ (XY model), where the action variables $J_{1}, J_{2}$ are related to the $J_{1}^{\prime}, J_{2}^{\prime}$ from (V.15) by a linear transformation. That transformation is to be constructed by the requirement that for any particular choice of line integrals (V.9) the quantum numbers $m_{1}, m_{2}$ can be assigned according to (IV.1) [25]. The 

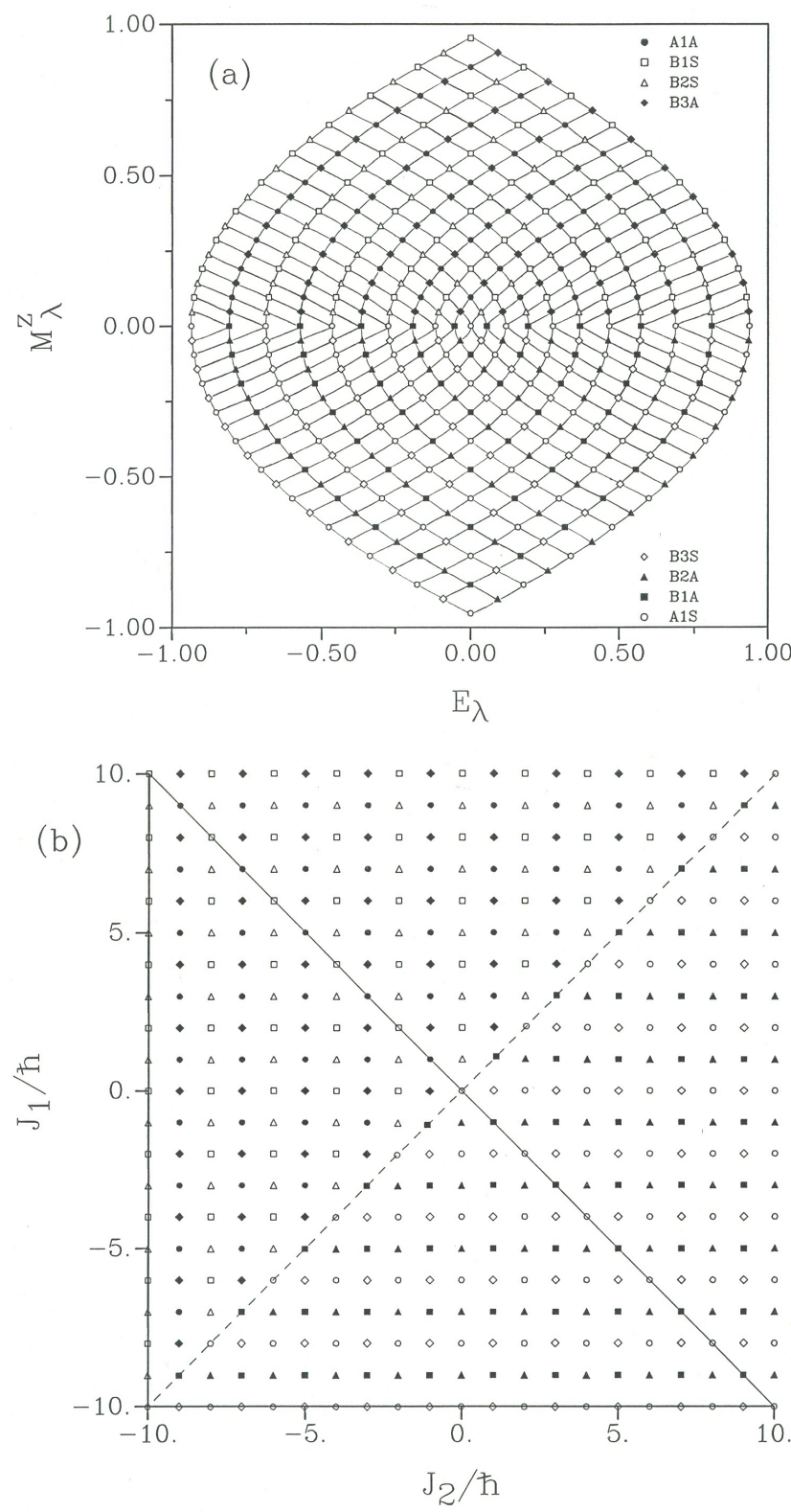

FIG. 4: (a) Eigenvalue $M_{\lambda}^{z}$ (magnetization) versus eigenvalue $E_{\lambda}$ (energy) of the $(2 \sigma+1)^{2}=441$ eigenstates of the quantum 2-spin $X Y$ model, (V.13) with $J_{z}=0, J=1$, for $\sigma=10$ and $s=1$. Same data as in Fig. 3(a). (b) Images of these eigenstates in the action plane $\left(J_{1}, J_{2}\right)$ as provided by the transformation (V.15) followed by a linear transformation. The solid (dashed) diagonal in the action plane corresponds to the line $E=0\left(M_{z}=0\right)$ in the $\left(E, M_{z}\right)$-plane. The eight different symbols label eight different symmetry classes of eigenstates as explained in the text.

pattern of points in the $\left(E, M_{z}\right)$-plane shown in Fig. 4(a) then maps onto an almost perfect square-lattice of size $(2 \sigma+1) \times(2 \sigma+1)$ in the action plane as shown in Fig. 4(b). The predictions of semiclassical quantization are borne out within the expected accuracy, viz.
$\mathrm{O}\left(\hbar^{2}\right)$ i.e. $\mathrm{O}\left(s^{2} / \sigma^{2}\right)$. The nearest-neighbor bonds of the square lattice [Fig. 4(b)] become the bonds drawn in Fig. 4(a). The eight different symbols used to characterize the points in Fig. 4 distinguish states belonging to eight different symmetry classes (to be discussed in Sect. VI).

The mapping $\left(M_{z}, E\right) \rightarrow\left(J_{1}, J_{2}\right)$ has a much simpler structure in the two cases for which the classical dynamics is linear. In the Heisenberg case $\left(J_{z}=J\right)$, both spins precess uniformly about the direction of the conserved vector $\mathbf{S}_{T}=\mathbf{S}_{1}+\mathbf{S}_{2}$; the precession rate is $\omega=\left|\mathbf{S}_{T}\right|$ for both spins. The energy and magnetization eigenvalues for all $(2 \sigma+1)^{2}$ eigenstates are:

$$
\begin{aligned}
E(l, m) & =\hbar^{2}[\sigma(\sigma+1)-l(l+1) / 2] \\
M_{z}(l, m) & =\hbar m / 2,
\end{aligned}
$$

where $l=0,1,2, \ldots, 2 \sigma$ is the quantum number of the total spin, and $m=-l,-l+1, \ldots, l$ its $z$-component. In this case, the prescription (IV.1) of semiclassical quantization is satisfied exactly by the variables $J_{1}=\hbar[\sigma-l+$ $\max (m, 0)], J_{2}=\hbar[\sigma-l+\min (m, 0)]$. It immediately follows that one of the two frequencies of the time evolution, $\omega_{1}=\partial H / \partial J_{k}, k=1,2$, is equal to $l$ and the other one is zero, in accordance with our analysis of the classical motion $[17,18]$. In the Ising case $(J=0)$, the situation is even simpler; here the canonical variables (II.7) are already action-angle variables. Setting $p_{k}=J_{k}=\hbar m_{k}$, $k=1,2, m_{k}=-\sigma,-\sigma+1, \ldots, \sigma$, the coordinates of the eigenstates in the $\left(E, M_{z}\right)$-plane are

$$
\begin{aligned}
E_{m_{1}, m_{2}} & =-\hbar^{2} m_{1} m_{2}, \\
M_{m_{1}, m_{2}}^{z} & =\hbar\left(m_{1}+m_{2}\right) / 2 .
\end{aligned}
$$

The two frequencies are $m_{1}$ and $m_{2}$.

\section{INTEGRABLE MODEL, NONSEPARABLE COORDINATES}

Here we consider the $X Y$ model with exchange anisotropy,

$$
\hat{H}_{\gamma}=-\hbar^{2}\left[(1+\gamma) \hat{\sigma}_{1}^{x} \hat{\sigma}_{2}^{x}+(1-\gamma) \hat{\sigma}_{1}^{y} \hat{\sigma}_{2}^{y}\right],
$$

the quantum version of (III.5). The integrability condition (II.9) is satisfied for arbitrary $\gamma$. Starting out with the isotropic $X Y$ model as in Sect. V, we now add a perturbation which breaks the continuous rotational symmetry. No separable canonical coordinates have been found for an exact analysis parallel to that of Sect V.

\section{A. Discrete symmetries}

The symmetry group of the general 2-spin Hamiltonian (II.4), of which $\hat{H}_{\gamma}$ is a special case, is $D_{2} \otimes I \otimes S_{2}$ [26], where $I$ is the inversion in spin space and $S_{2}=(E, P)$ the permutation group of the two spins. $D_{2}$ contains all the 
twofold rotations about the coordinate axes. For spins the inversion is equivalent to time reversal. Therefore, if all irreducible representations of the group are real, as is the case here, it is sufficient to consider the group $D_{2} \otimes S_{2}$, whose characters are reproduced in Table I. The eigenvectors of $\hat{H}$ can thus be classified according to eight different symmetry classes with transformation properties corresponding to the eight different irreducible representations of $D_{2} \otimes S_{2}$. The explicit incorporation of these discrete symmetries into the calculation is pivotal for the interpretation of quantum invariants.

\begin{tabular}{|c|c|c|c|c|c|c|c|c|}
\hline$D_{2} \otimes S_{2}$ & $E$ & $C_{2}^{z}$ & $C_{2}^{y}$ & $C_{2}^{x}$ & $P$ & $P C_{2}^{z}$ & $P C_{2}^{y}$ & $P C_{2}^{x}$ \\
\hline A1S & 1 & 1 & 1 & 1 & 1 & 1 & 1 & 1 \\
\hline A1A & 1 & 1 & 1 & 1 & -1 & -1 & -1 & -1 \\
\hline B1S & 1 & 1 & -1 & -1 & 1 & 1 & -1 & -1 \\
\hline B1A & 1 & 1 & -1 & -1 & -1 & -1 & 1 & 1 \\
\hline $\mathrm{B} 2 \mathrm{~S}$ & 1 & -1 & 1 & -1 & 1 & -1 & 1 & -1 \\
\hline B2A & 1 & -1 & 1 & -1 & -1 & 1 & -1 & 1 \\
\hline B3S & 1 & -1 & -1 & 1 & 1 & -1 & -1 & 1 \\
\hline B3A & 1 & -1 & -1 & 1 & -1 & 1 & 1 & -1 \\
\hline
\end{tabular}

TABLE I: Character table of the symmetry group $D_{2} \otimes S_{2}$, where $D_{2}$ contains all twofold rotations $C_{2}^{\alpha}, \alpha=x, y, z$ about the coordinate axes and $S_{2}=(E, P)$ is the permutation group. The dimensionalities of the invariant subspaces in the $(2 \sigma+1)^{2}$-dimensional Hilbert space of the Hamiltonian (II.4) for integer-valued $\sigma$ are $(2 \sigma+2)(2 \sigma+4) / 8$ for A1S states, $2 \sigma(2 \sigma-2) / 8$ for AIA states and $2 \sigma(2 \sigma+2) / 8$ for any of the other six symmetry classes of states.

In Sect. V, specifically in Figs. 3 and 4(a), we have used eigenstates which are common to the two commuting operators $\hat{M}, \hat{M}_{z}$ for the determination of quantum invariants. However, $\hat{M}_{z}$ is not invariant under all transformations of $D_{2} \otimes S_{2}$ and the matrix elements $\left\langle\lambda\left|\hat{M}_{z}\right| \lambda\right\rangle$ vanish identically for all symmetry-adapted eigenvectors of $\hat{H}$. If we want to recover Fig. 4(a) by use of symmetryadapted eigenvectors, we must consider instead the matrix elements $\sqrt{\left\langle M_{z}^{2}\right\rangle_{\lambda}}= \pm \sqrt{\left\langle\lambda\left|\hat{M}_{z}^{2}\right| \lambda\right\rangle}$ and assign the minus sign (somewhat artificially) to four out of eight symmetry classes. Figure 4(a) represents a particular choice.

\section{B. The spider's web}

For the isotropic $X Y$ model $\hat{H}_{\gamma=0}$, the states of all eight symmetry classes for a single pattern in the $\left(E, M_{z}\right)$-plane [see Fig. 4(a)], and that pattern transforms as a whole under a uniaxial perturbation (see Fig. 3). This is no longer the case for any biaxial perturbation. Upon increasing the amount of exchange anisotropy in the $X Y$ model (VI.1) from $\gamma=0$ to $\gamma=0.2$, the single pattern of points in the $\left(E, \sqrt{\left\langle M_{z}^{2}\right\rangle}\right)$ plane shown in Fig. 4(a) breaks up, as depicted in Fig. 5, into four different sheets, each containing states of two
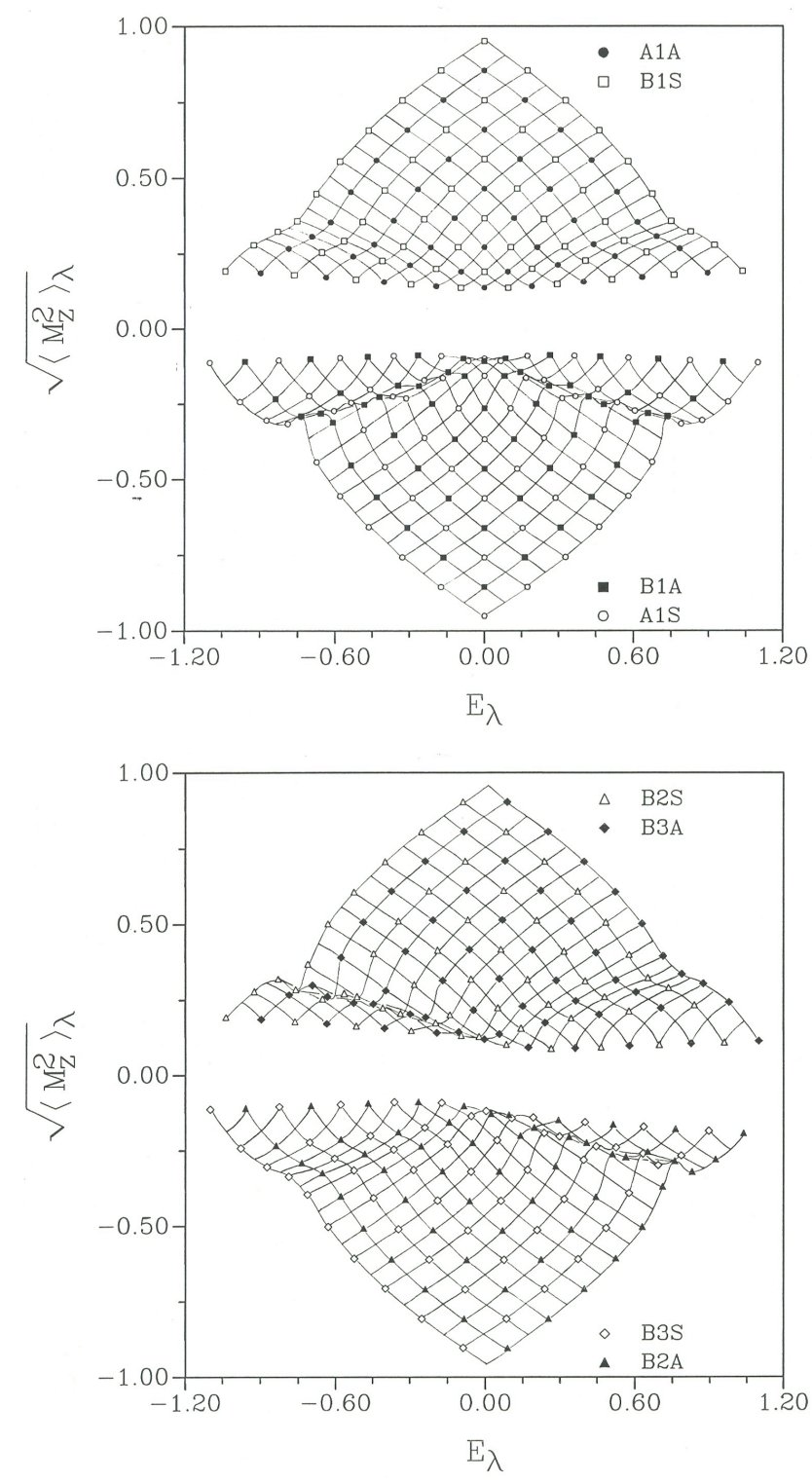

FIG. 5: Invariant $\sqrt{\left\langle M_{z}^{2}\right\rangle_{\lambda}}= \pm \sqrt{\left\langle\lambda\left|\hat{M}_{z}^{2}\right| \lambda\right\rangle}$ versus eigenvalue $E_{\lambda}$ (energy) for the eigenstates of the quantum 2-spin $X Y$ model (VI.1) with $\gamma=0.2$ and for $\sigma=10, s=1$. The four different plots are for states with different transformation properties under the discrete symmetry group $D_{2} \otimes S_{2}$. The points are connected to a web in the same way as in Fig. 4(a) for the isotropic case $(\gamma=0)$.

symmetry classes. The signature of integrability is still fully present in these patterns. In each of the four $\left(E, \sqrt{\left\langle M_{z}^{2}\right\rangle}\right)$-sheets the states can be naturally connected to a fully intact $2 d$ web with coordination number 4 . These webs are graphical representations of the nonlinear transformation between the two analytic invariants $E, \sqrt{\left\langle M_{z}^{2}\right\rangle}$ and the two action variables $J_{1}, J_{2}$. The existence of that transformation, though not explicitly known, is guaranteed. Each point on the $\left(J_{1}, J_{2}\right)$-plane 
and its image in the $\left(E, \sqrt{\left\langle M_{z}^{2}\right\rangle}\right)$-plane represent an invariant torus. In the classical system, these tori densely foliate the entire phase space, whereas, upon quantization, each torus claims a nonzero volume, $\Delta \Omega=\hbar^{2}$, in accordance with the uncertainty principle.

Connecting the points in the $\left(E, \sqrt{\left\langle M_{z}^{2}\right\rangle}\right)$-plane by bonds until they form a complete web with 4 bonds per vertex, amounts to assigning a pair of quantum numbers to each eigenstate. There is some arbitrariness in the construction of the web. In Fig. 5 we have chosen four webs, each consisting of two interpenetrating components. Each component is populated by states of one symmetry class. Alternatively, a one-component web of smaller mesh size can be drawn through the points of each pair of symmetry classes (to be used in subsequent figures). In either case, we can interpret the threads of the web as lines of constant action and the meshes along one such line as marks of increments in units of $\hbar$ for the other action variable. Different webs thus represent different choices of action variables, which are related to one another by linear transformations. This freedom of choice in picking a pair of action variables or assigning pairs of quantum numbers also alerts us to the fact that the notion of "neighboring tori" is much more ambiguous in $4 d$ phase space than our $3 d$-anchored intuition might suggest.

In order to establish the connection between this quantum invariant-web and the classical invariant-surface

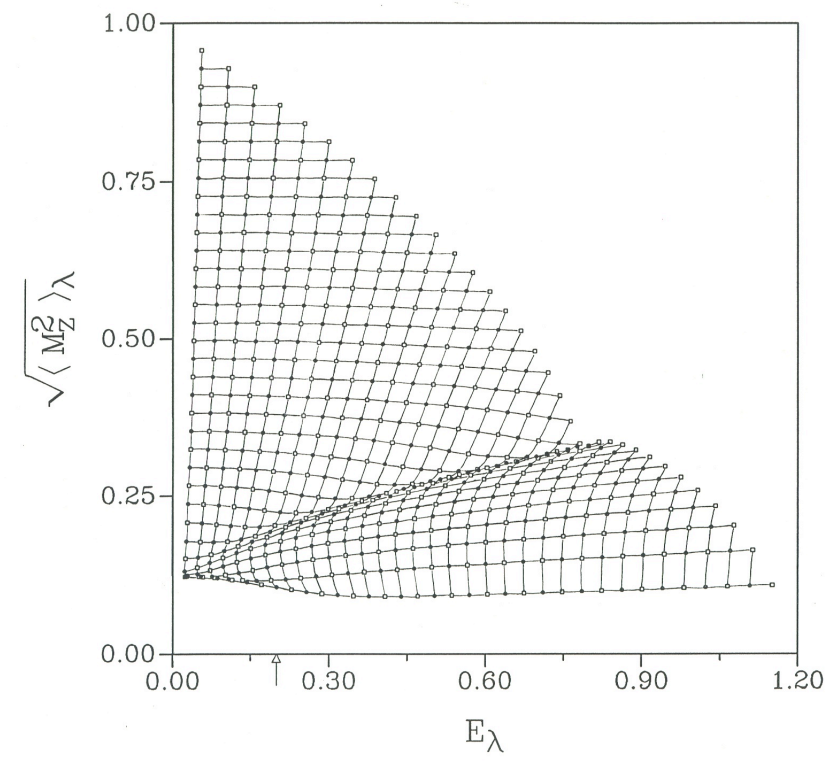

FIG. 6: Invariant $\sqrt{\left\langle M_{z}^{2}\right\rangle_{\lambda}}=\sqrt{\left\langle\lambda\left|\hat{M}_{z}^{2}\right| \lambda\right\rangle}$ versus eigenvalue $E_{\lambda}$ (energy) at $E>0$ for class A1A states (full circles) and B1S states (open squares) of the integrable quantum 2-spin $X Y$ model (VI.1) for $\sigma=35, s=1$ and for the value $\gamma=$ 0.2 of the anisotropy parameter. The total number of states shown is 595 .
$E\left(\sqrt{\left\langle M_{x}^{2}\right\rangle}, \sqrt{\left\langle M_{z}^{2}\right\rangle}\right)$ discussed in Sect. III.1, we must interpret the 4 patterns in Fig. 5 as projections onto the $\left(E, \sqrt{\left\langle M_{z}^{2}\right\rangle}\right)$-plane of 4 individual $2 d$ webs in $3 d$ $\left(E, \sqrt{\left\langle M_{x}^{2}\right\rangle}, \sqrt{\left\langle M_{z}^{2}\right\rangle}\right)$-space, one for each of the 4 symmetry classes of eigenstates. The 4 discrete functions, $E_{\lambda}^{(n)}\left(\sqrt{\left\langle M_{x}^{2}\right\rangle_{\lambda}}, \sqrt{\left\langle M_{z}^{2}\right\rangle_{\lambda}}\right), n=1, \ldots, 4$, can each be interpolated by a continuous function, and the average of these functions becomes, in the classical limit (II.5), the function $E\left(\sqrt{\left\langle M_{x}^{2}\right\rangle}, \sqrt{\left\langle M_{z}^{2}\right\rangle}\right)$, which describes the classical invariant-surface.

For an illustration of this connection, we show in Fig. 6 the web for A1A and B1S states with $E>0$ of $\hat{H}_{\gamma=0.2}$ with $\sigma=35$, again in a projection onto the $\left(E, \sqrt{\left\langle M_{z}^{2}\right\rangle}\right)$ plane [27]. The arrow indicates the energy at which the corresponding classical invariant-surface is intersected for presentation in Fig. 1. Note how the cusp singularities in the classical invariant-surface section are represented in the quantum invariant-web by smooth folds. These folds become more and more pronounced as $\sigma$ increases.

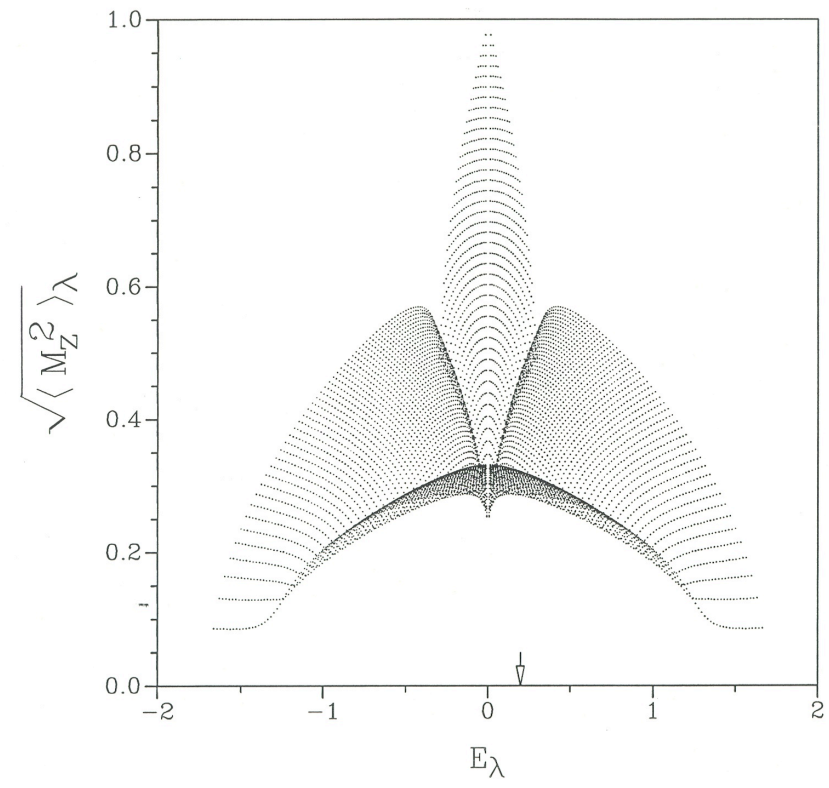

FIG. 7: Invariant $\sqrt{\left\langle M_{z}^{2}\right\rangle_{\lambda}}=\sqrt{\left\langle\lambda\left|\hat{M}_{z}^{2}\right| \lambda\right\rangle}$ versus eigenvalue $E_{\lambda}$ (energy) at $E \neq 0$ for classes $\mathrm{A} 1 \mathrm{~A}$ and B1S states of the integrable quantum 2-spin model $\hat{H}_{\gamma=0.7}$ for $\sigma=90, s=1$. The total number of states shown is 8010 .

An important feature of the classical time evolution of $H_{\gamma}$ is the existence for $\gamma>0$ of separatrices in phase space. In some cases, they can be easily located in the $\left(E, \sqrt{\left\langle M_{z}^{2}\right\rangle}\right)$-web. In Fig. 6, for example, one set of threads change from positive to negative slope going through a point of infinite slope if the quantum states are smoothly interpolated. Interpreting these lines as lines of constant action, say $J_{2}=$ const, it follows that one of two fundamental frequencies of the classical time evolution, $\omega_{1}=\partial H^{\prime}\left(J_{1}, J_{2}\right) / \partial J_{1}$, slows down to zero at the point of infinite slope, which signals the presence of a separatrix 
in the action plane. Our particular choice of web makes it easy to locate one separatrix line through the web shown in Fig. 6.

All the observations made thus far are beautifully illustrated by Fig. 7, which depicts an invariant-web for $\hat{H}_{\gamma=0.7}$ in the same representation. Plotted are all 8010 A1A and B1S states at $E \neq 0$ for $\sigma=90$, which form a highly regular pattern. This pattern is highly suggestive of a smooth surface in $\left(E, \sqrt{\left\langle M_{x}^{2}\right\rangle}, \sqrt{\left\langle M_{z}^{2}\right\rangle}\right)$-space, parts of which project two or three layers onto the $\left(E, \sqrt{\left\langle M_{z}^{2}\right\rangle}\right)$ plane. That surface, cut at $E=0.2$ (see arrow), connects well with the section of the classical invariant-surface depicted in Fig. 1. Note particularly that the fabric of the web is fully intact even in the immediate vicinity of a separatrix. In the nonintegrable model (to be discussed next), this is the location where chaos makes its appearance most conspicuously.

\section{NONINTEGRABLE MODEL}

Starting out again with the isotropic $X Y$ model (as in Sects. V and VI) we now add a perturbative term to the Hamiltonian which breaks the continuous rotational symmetry and destroys the integrability. Consider the 2-spin Hamiltonian

$$
\begin{aligned}
\hat{H}_{\alpha}= & -\hbar^{2}\left\{\hat{\sigma}_{1}^{x} \hat{\sigma}_{2}^{x}+\hat{\sigma}_{1}^{y} \hat{\sigma}_{2}^{y}\right. \\
& \left.+\frac{1}{2} \alpha\left[\left(\hat{\sigma}_{1}^{x}\right)^{2}-\left(\hat{\sigma}_{1}^{y}\right)^{2}+\left(\hat{\sigma}_{2}^{x}\right)^{2}-\left(\hat{\sigma}_{2}^{y}\right)^{2}\right]\right\},
\end{aligned}
$$

whose classical counterpart (III.6) is manifestly chaotic for $\alpha \neq 0, \pm 1[18]$. We shall demonstrate that the quantum invariant-web is affected by the presence of chaos in a way which is in direct relation to our observations made in Sect. III for the classical invariant-surface.

\section{A. The fly in the spider's web}

Figure 8(a) shows the quantum invariant-web for the case of a relatively weak nonintegrable perturbation $(\alpha=$ $0.35)$ of this model. Plotted are the states A1A and B1S for $\sigma=35$. This should be compared with Fig. 6, in which the corresponding web for a weak integrable perturbation $(\gamma=0.2)$ with the same symmetry is plotted. In this classically nonintegrable case, the web is fully intact as if the model were integrable. All points $\left(E_{\lambda}, \sqrt{\left\langle M_{z}^{2}\right\rangle_{\lambda}}\right)$ can be associated with quantized tori, which form a coherent network seemingly unimpeded by the dense chaotic phase flow between the intact classical tori. As long as chaotic trajectories are confined to regions of the action plane which are much smaller than the mesh size of the invariant-web, the assignment of quantum-number pairs to the states is as natural and straightforward as for the integrable model $\hat{H}_{\gamma}$ (Fig. 6).

However, in the case of $\hat{H}_{\alpha}$ [Fig. 8(a)], we know that these quantum numbers do not represent global action
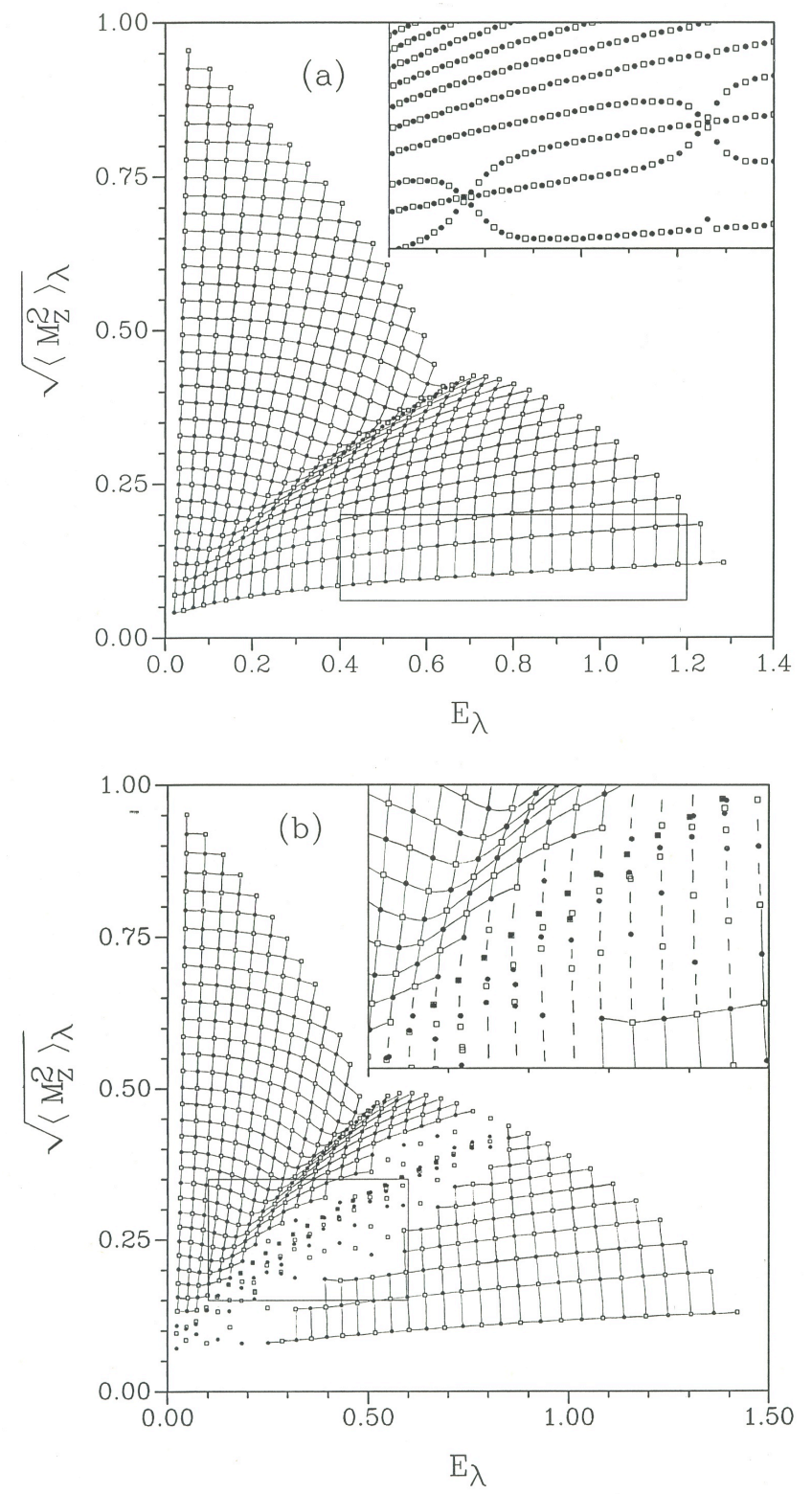

FIG. 8: Invariant $\sqrt{\left\langle M_{z}^{2}\right\rangle_{\lambda}}= \pm \sqrt{\left\langle\lambda\left|\hat{M}_{z}^{2}\right| \lambda\right\rangle}$ versus eigenvalue $E_{\lambda}$ (energy) at $E>0$ for class A1A states (full circles) and B1S states (opens squares) of the nonintegrable quantum 2spin $X Y$ model (VII.1) for $\sigma=35, s=1$ and for values (a) $\alpha=0.35$, (b) $\alpha=0.5$ of the anisotropy parameter. The inset to (a) shows the framed part of the $\left(E, \sqrt{\left\langle M_{z}^{2}\right\rangle}\right)$-plane for the same model but with $\sigma=90$. The inset to (b) shows the framed part of the web magnified.

variables of the classical model $H_{\alpha}$, because the latter do not exist. When we decrease the mesh size of the web by increasing the value of $\sigma$, we shall see that beyond some threshold, the fabric of the web starts to fall apart. Knowing that the tori are nowhere dense in phase space, we must conclude that no part of the web will stay intact close enough to the classical limit. 
From our analysis in [18] of the classical model (III.6), we know that a separatrix line should be present near the edge at low values of $\sqrt{\left\langle M_{z}^{2}\right\rangle}$ in the quantum invariantweb of $\hat{H}_{\alpha=0.35}$ [Fig. $8(\mathrm{a})$ ], similar to the one identified for $\hat{H}_{\gamma=0.2}$ (Fig. 6). However, in the nonintegrable case, it is the quantum image of a narrow chaotic band in phase space. For $\sigma=35$, the case shown in Fig. 8(a), no evidence for a separatrix line can be discerned. Unlike in Fig. 6, none of the lines of constant action go through a point of infinite slope upon smooth interpolation. Quantum mechanics has pushed the separatrix line towards lower values of $\sqrt{\left\langle M_{z}^{2}\right\rangle}$ into the kinematically inaccessible region. But when we analyze the invariant-web of the same model, $\hat{H}_{\alpha=0.35}$, closer to the classical limit, we observe dramatic changes in the arrangement of quantum states. The inset to Fig. 8(a) reproduces the framed part of the invariant-web for a higher spin quantum number $(\sigma=90)$. Now the separatrix line has moved into the kinematically accessible region, as in Fig. 6. But in the nonintegrable model, nearly degenerate states (of the same symmetry class) tend to resonate in the vicinity of the separatrix line. In the inset to Fig. 8(a) we can clearly identify two strong resonances and a weak one.

For the same model at still higher $\sigma$-values, more and more such resonances are expected to make their appearance in the vicinity of the separatrix line. As their number increases, these resonances begin to overlap. The overlap of resonances was introduced by Chirikov [28] as a criterion for widespread chaos in the context of classical perturbation theory, and quantum analogs of that concept have since been used in a number of different applications [29, 30].

Instead of increasing $\sigma$, we produce essentially the same effect by increasing $\alpha$ to make the nonintegrable perturbation stronger and thus generate a wider band of chaos along the separatrix in the classical phase flow. Figure $8(\mathrm{~b})$ shows the quantum invariant-web of $\hat{H}_{\alpha=0.4}$ for $\sigma=35$. Here no individual resonances can be identified any more. The fabric of the web is now destroyed beyond repair in the vicinity of the separatrix line. Within the chaotic region, the quantum states tend to cluster in short strips along lines which interpolate one set of threads of the web across the separatrix line, leaving sizable areas of the $\left(E, \sqrt{\left\langle M_{z}^{2}\right\rangle}\right)$-plane almost depleted of states. The inset to Fig. 8(b) shows a close-up view of the web in and around the chaotic region. Here we can discern how the quantum states within one of these short strips in the chaotic region are slightly displaced sideways parallel to the energy axis. This is the well-known phenomenon of level repulsion. In this representation, the effect appears to be minute, but after projection of the points onto the $E$-axis, these sideways displacements are, in fact, of the same order of magnitude as the mean level spacing [31].

\section{B. The spider returns}

Between $\alpha=0.5$ and $\alpha=0.7$, the chaotic region around the separatrix has increased considerably in size. Here (at $\alpha=0.7$ ) a new feature of major importance makes its appearance in the quantum invariant-web, as can be observed in Fig. 9. Shown are the quantum invariants $E_{\lambda}, \sqrt{\left\langle M_{z}^{2}\right\rangle_{\lambda}}$ for all A1A and B1S eigenstates of $\hat{H}_{\alpha=0.7}$ for $\sigma=45$ (within a window of given size). The arrow indicates the energy at which the remnant of the corresponding classical invariant-surface is intersected for presentation in Fig. 2.

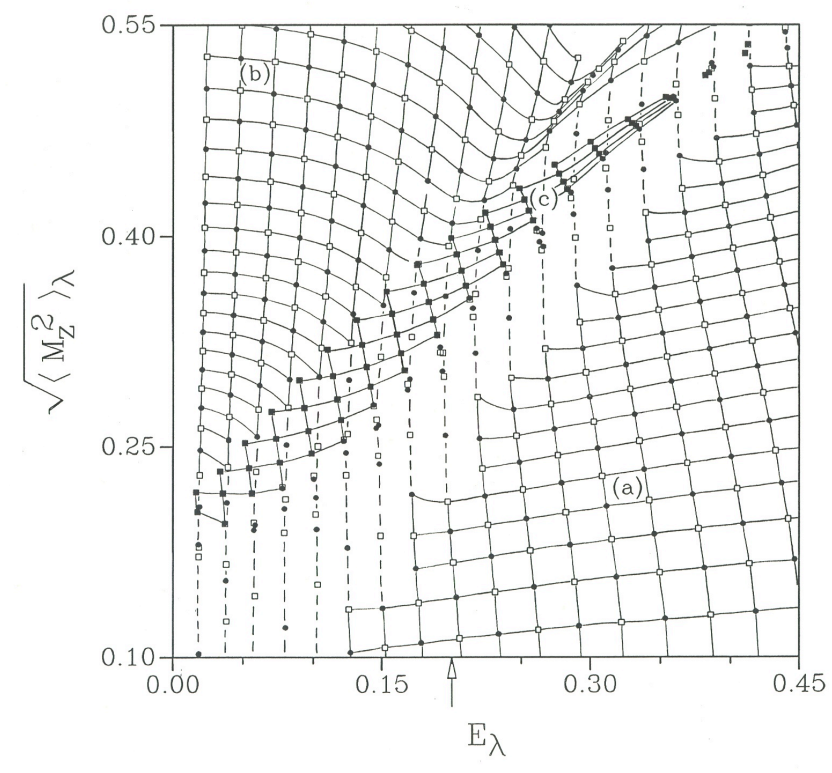

FIG. 9: Invariant $\sqrt{\left\langle M_{z}^{2}\right\rangle_{\lambda}}= \pm \sqrt{\left\langle\lambda\left|\hat{M}_{z}^{2}\right| \lambda\right\rangle}$ versus eigenvalue $E_{\lambda}$ (energy) at $E>0$ for all eigenstates of symmetry classes A1A (full circles) and B1S (open squares) of the nonintegrable quantum 2 -spin model $\hat{H}_{\alpha=0.7}$ with spin quantum number $\sigma=45$ and $s=1$. Only states within a certain window of values for $\sqrt{\left\langle M_{z}^{2}\right\rangle_{\lambda}}$, and $E_{\lambda}$ are shown.

The correspondence between classical and quantum nonintegrability effects in this representation is indeed quite remarkable. In the regular regions at top left and bottom right, the quantum web is fully intact; here chaos is confined to areas much smaller than the mesh size. Between the two regular regions extends a broad band of chaos along a separatrix of the classical motion. Here the quantum web is interrupted, as already observed in Fig. 8(b) for the case $\alpha=0.5$, and the quantum states again tend to cluster in short strips along the dashed lines. However, superimposed onto the region populated by the chaotic states (in the projection of Fig. 9), we now find a new web of quantized type (c) tori, a web which is disconnected from the primary web and which has its classical counterpart in the secondary KAM tori discussed in the context of Fig. 2. Note also that in the 
projection of Fig. 9, the A1A and B1S states avoid each other in regions (a) and (b), whereas they lie on top of one another in region (c). This further underlines the different character of primary and secondary KAM tori.

In summary, the classical and quantum invariants as represented in this study, provide a novel direct link between manifestations of Hamiltonian chaos and quantum nonintegrability effects. The structure of the Hamiltonian phase flow has its precise image in the classical invariant-surface and an image of finite resolution in the quantum invariant-web. The destruction of invariant tori is paralleled by the disappearance of quantum numbers and the restoration of secondary KAM tori by the reappearance of new quantum numbers.

\section{Acknowledgments}

We thank Charles Kaufman for useful comments and suggestions. This work was supported in part by the US National Science Foundation, Grant DMR-90-07540 and by Sigma Xi, the Scientific Research Society. The numerical calculations were performed on the CRAY-2 of the National Center for Supercomputing Applications, University of Illinois at Urbana-Champaign.

\section{Appendix A: Exact solution of the classical 2-spin $x x z$ model}

Here we outline the general solution for the time evolution of the classical 2-spin model

$$
H=-J\left(S_{1}^{x} S_{2}^{x}+S_{1}^{y} S_{2}^{y}\right)-J_{z} S_{1}^{z} S_{2}^{z}
$$

studied in Sect. V. In terms of spherical coordinates (II.1), the four equations of motion read

$$
\begin{aligned}
& \dot{\vartheta}_{1}=s J \sin \vartheta_{2} \sin \left(\varphi_{1}-\varphi_{2}\right) \\
& \dot{\varphi}_{1}=s J \cot \vartheta_{1} \sin \vartheta_{2} \cos \left(\varphi_{1}-\varphi_{2}\right)-J_{z} s \cos \vartheta_{2}
\end{aligned}
$$

and $1 \leftrightarrow 2$. Introducing two new variables

$$
z=s\left(\cos \vartheta_{1}-\cos \vartheta_{2}\right) / 2, \quad \zeta=\tan \left(\varphi_{1}-\varphi_{2}\right)
$$

and using the two integrals of the motion

$$
\begin{gathered}
E=-J s^{2} \sin \vartheta_{1} \sin \vartheta_{2} \cos \left(\varphi_{1}-\varphi_{2}\right) \\
-J_{z} s^{2} \cos \vartheta_{1} \cos \vartheta_{2} \\
M_{z}=s\left(\cos \vartheta_{1}+\cos \vartheta_{2}\right)
\end{gathered}
$$

we can combine (A.2) to a set of two $1^{\text {st }}$ order ODEs,

$$
\begin{aligned}
& \dot{z}=\zeta\left[E+J_{z}\left(M_{z}^{2}-z^{2}\right)\right], \\
& \dot{\zeta}=-\frac{2 z J^{2}\left(s^{2}+M_{z}^{2}-z^{2}\right)}{E+J_{z}\left(M_{z}^{2}-z^{2}\right)}+2 J_{z} z\left(1+\zeta^{2}\right),
\end{aligned}
$$

which in turn can be formulated as Newton's equation of motion, $\ddot{z}=-V^{\prime}(z)$, for a fictitious particle of unit mass in a $1 d$ anharmonic potential

$$
V(z)=\left[J^{2} s^{2}-J_{z} E+\left(J^{2}-J_{z}^{2}\right) M_{z}^{2}\right] z^{2}-\frac{1}{2}\left(J^{2}-J_{z}^{2}\right) z^{4} .
$$

For $J>J_{z}, V(z)$ has the shape of an inverted double-well with a local minimum at $z=0$ and maxima at $z= \pm z_{m}$, where

$$
z_{m}^{2}=M_{z}^{2}+\frac{J^{2} s^{2}-J_{z} E}{J^{2}-J_{z}^{2}},
$$

and the particle motion is kinematically constrained to bounded motion. For the case of the Heisenberg model $\left(J=J_{z}\right)$, the potential becomes harmonic, $V(z)=$ $J\left(s^{2}-E\right) z^{2}$. For $J<J_{z}$, the shape of $V(z)$ undergoes a qualitative change as a function of the parameter $E$ (energy of spin system). For low values of $E$, we have $z_{m}^{2}<0$, and $V(z)$ is a single-well potential with a minimum at $z=0$. As $E$ increases, $V(z)$ undergoes a transition (at $\left.z_{m}^{2}=0\right)$ to a double-well potential with a local maximum at $z=0$ and minima at $z= \pm z_{m}$.

For an explicit solution of this simple dynamical problem, we calculate the particle energy, a conserved quantity, in terms of the invariants $E, M_{z}$ :

$$
U\left(M_{z}, E\right)=\frac{1}{2}\left[J^{2}\left(s^{2}-M_{z}^{2}\right)^{2}-\left(E+J_{z} M_{z}^{2}\right)^{2}\right]=\text { const. }
$$

The function $z(t)$ is then obtained from the integral

$$
t=\int_{z_{0}}^{z} \frac{d z^{\prime}}{\sqrt{2\left[U-V\left(z^{\prime}\right)\right]}}
$$

with the turning point $z_{0}$ determined from the roots of $V(z)=U$. The function $z(t)$ in conjunction with the invariants (A.4-5) describes the meridional motion of the two spins completely. The azimuthal motion, on the other hand, is described by the function $\zeta(t)$, expressible by (A.6) in terms of $z(t)$ and $\dot{z}(t)$, and by the integral of

$$
\begin{aligned}
\dot{\Phi} & \equiv \frac{\mathrm{d}}{\mathrm{d} t} \frac{1}{2}\left(\varphi_{1}+\varphi_{2}\right) \\
& =-\frac{2 M_{z} J^{2}\left(s^{2}-M_{z}^{2}+z^{2}\right)}{\left[E+J_{z}\left(M_{z}^{2}-z^{2}\right)\right]\left(1+\zeta^{2}\right)}-2 J_{z} M_{z} .
\end{aligned}
$$

The solution used in Sect. V for the evaluation of action variables corresponds to the situation $J>J_{z}$. Here we have

$$
\begin{aligned}
& z(t)=z_{0} \operatorname{sn}\left(a t, \frac{z_{0}}{a}\right), \\
& \zeta(t)=\frac{a z_{0} \operatorname{cn}\left(a t, \frac{z_{0}}{a}\right) \operatorname{dn}\left(a t, \frac{z_{0}}{a}\right)}{E+J_{z}\left[M_{z}^{2}-z_{0}^{2} \operatorname{sn}^{2}\left(a t, \frac{z_{0}}{a}\right)\right]},
\end{aligned}
$$

where $z_{0}^{2}=z_{m}^{2}-\sqrt{z_{m}^{4}-c}, a^{2}=z_{m}^{2}+\sqrt{z_{m}^{4}-c}$ and $c=\left[J^{2}\left(s^{2}-M_{z}^{2}\right)^{2}-\left(E+J_{z} M_{z}^{2}\right)^{2}\right] /\left(J^{2}-J_{z}^{2}\right)$. The two 
fundamental frequencies of this dynamical system are determined by the following expressions:

$$
\tau_{2}\left(M_{z}, E\right)=\frac{4}{a} \mathrm{~K}\left(z_{0} / a\right)=2 \pi / \omega_{2}\left(M_{z}, E\right)
$$

$$
\omega_{1}\left(M_{z}, E\right)=\frac{1}{\tau_{2}} \int_{0}^{\tau_{2}} \mathrm{~d} t \dot{\Phi}(t)=2 \pi / \tau_{1}\left(M_{z}, E\right)
$$

[1] Lichtenberg, A.J., Lieberman, M.A.: Regular and stochastic motion. Berlin, Heidelberg, New York: Springer 1983.

[2] Tabor, M.: Chaos and integrability in nonliner dynamics. New York: Wiley 1989.

[3] Sagdeev, R.Z., Usikov, D.A., Zaslavsky, G.M.: Nonlinear physics. From the pendulum to turbulence and chaos. Chur: Harwood 1988.

[4] Ozorio de Almeida, A.M.: Hamiltonian systems. Chaos and quantization. Cambridge: Cambridge University Press 1988.

[5] Wolfram, S. : Phys. Rev. Lett. 55, 449 (1985).

[6] Ford, J.: Phys. Today 33, No. 4, 40 (1983).

[7] Chaitin, G.J.: Algorithmic information theory. Cambridge: Cambridge University Press 1987.

[8] Chaotic behavior in quantum systems. Theory and applications. Casati, G. (ed.). New York: Plenum Press 1985.

[9] Quantum measurement and chaos. Pike, E.R., Sarkar, S. (eds.). New York: Plenum Press 1987.

[10] Eckhardt, B.: Phys. Rep. 163, 205 (1988).

[11] Bohigas, O., Giannoni, M.J., Schmit, C.: Phys. Rev. Lett. 52, 1 (1984).

[12] José, J.V.: In: Directions in chaos. Bai-Lin, H. (ed.), Vol. II. Singapore:World Scientific 1989.

[13] Van Leeuwen, K.A.H., et al.: Phys. Rev. Lett. 55, 2231 (1985).

[14] Bardsley, J.N., et al.: Phys. Rev. Lett. 56, 1007 (1986).

[15] Holle, A., et al. : Phys. Rev. Lett. 56, 2594 (1986).

[16] Hoffnagle, J., De Voe, R.G., Reyna, L., Brewer, R.G.: Phys. Rev. Lett. 61, 255 (1988).

[17] Magyari, E., Thomas, H., Weber, R., Kaufman, C., Müller, G.: Z. Phys. B - Condensed Matter 65, 363 (1987).

[18] Srivastava, N., Kaufman, C., Müller, G., Weber, R., Thomas, H.: Z. Phys. B - Condensed Matter 70, 251 (1988).

[19] Srivastava, N., Kaufman, C., Müller, G.: J. Appl. Phys.
63, 4154 (1988).

[20] Srivastava, N., Kaufman, C., Müller, G.: J. Phys. (Paris) C8, 1601 (1988).

[21] Khinchin, A.I. : Mathematical foundations of statistical mechanics. p. 19. New York: Dover 1949; Abraham, R., Marsden, J.E.: Foundation of mechanics, $2^{\text {nd }}$ Edn., p. 238. Reading: Benjamin/Cummings 1985.

[22] See Fig. 6 of Ref. 18 for an illustration.

[23] This representation of nonanalytic invariants was amply illustrated in Figs. 7 and 8 of Ref. 18.

[24] Peres, A.: Phys. Rev. Lett. 53, 1711 (1984).

[25] For the realization (V.15) of the line integral (V.9b), the appropriate choice of linear transformation which accomplishes this for $J_{z}=0$ is the following for symmetry classes AIS, A1A, B2S, B2A: $J_{1}=J_{1}^{\prime}+J_{2}^{\prime}-s, J_{1}=J_{2}^{\prime}-s$ if $J_{2}^{\prime}>0 ; J_{1}=J_{2}^{\prime}+s, J_{2}=J_{2}^{\prime}-J_{1}^{\prime}+s$ if $J_{2}^{\prime}<0$. For symmetry classes B1S, BIA, B3S, B3A interchange $J_{1}$ and $J_{2}$ in the above expression

[26] Weber, R. : Untersuchungen an klassischen Spinsystemen. Doctoral Thesis, University of Basel 1988, gives an exhaustive discussion of the symmetry properties and eigenvalue degeneracies of the 2-spin model (II.6).

[27] For simplicity we shall continue to refer to any single pattern in the $\left(E,\left\langle M_{z}^{2}\right\rangle\right)$-plane as one web even though in some cases it may only appear that way as a result of projection.

[28] Chirikov, B.V.: Phys. Rep. 52, 263 (1979).

[29] Berman, G.P., Zaslavskii, G.M.: Phys. Lett. 61A, 295 (1977); Berman, G.P., Zaslavskii, G.M., Kolovsky, A.R.: ibid. 87A, 152 (1982); Berman, G.P., Kolovsky, A.R. : ibid. 95A, 15 (1983).

[30] Lin, W.A., Reichl, L.E.: Phys. Rev. A 36, 5099 (1987); ibid. A 37, 3972 (1988).

[31] 31. Srivastava, N., Kaufman, C., Müller, G.: J. Appl. Phys. 67, 5627 (1990). 